'Servicio y Departamento de

Obstetricia, Ginecología y

Neonatología, Hospital San Borja Arriarán. Facultad de Medicina, Universidad de Chile, Santiago, Chile.

${ }^{2}$ Programa de Microbiología y Micología, Facultad de Medicina, Universidad de Chile, Santiago, Chile.

${ }^{3}$ Instituto de Investigaciones Materno Infantil (IDIMI), Facultad de Medicina, Universidad de Chile, Santiago, Chile. ${ }^{4}$ Servicio de Anatomía Patológica, Hospital San Borja Arriarán, Santiago, Chile.

Conflictos de interés: Ninguno.

Recibido el 10 de julio de 2015, aceptado el 5 de enero de 2016.

Correspondencia a: Alfredo Ovalle Salas Santa Rosa 1234 Santiago, Chile. Teléfono-fax: + 56-2-25555535 alfredoovalle@gmail.com

\section{Obesidad, factor de riesgo de infección bacteriana ascendente durante el embarazo}

\author{
ALFREDO OVALLE ${ }^{1}$, MARÍA ANGÉLICA MARTÍNEZ ${ }^{2}$, \\ ARIEL FUENTES ${ }^{3}$, XIMENA MARQUES ${ }^{1}$, FRANCISCO VARGAS ${ }^{1}$, \\ PAULA VERGARA ${ }^{1}$, PABLO STAIG ${ }^{1}$, MARÍA PAZ MARÍN ${ }^{1}$, \\ FRANCISCO ODA ${ }^{1}$, ELENA KAKARIEKA ${ }^{4}$
}

\section{Obesity, a risk factor for ascending bacterial infection during pregnancy}

Background: Obesity in pregnancy is associated with significantly higher rates of infection. Aim: To compare the infectious morbidity in pregnant women with normal and altered body mass index (BMI). Material and Methods: Cross sectional retrospective study of 6,150 patients who had delivery or second trimester abortion during 2012. The patients were classified according to BMI as underweight, normal weight, overweight and obese. We compared the frequency of pregnancy and perinatal complications related to ascending bacterial infection (ABI). The data was obtained from the hospital's databases. Results: Obese patients had higher rates of pregnancy and perinatal complications related to ABI compared to patients with normal weight. The odds ratios (OR) and 95\% confidence intervals (CI) for second trimester abortion were 3.45 (1.63-7.31) $p<0.01$, for preterm delivery $2.42(1.51-3.87) p<0.01$, for labor and puerperium infections $3.42(2.06-5.68) p<0.01$ and for early neonatal infectious and perinatal mortality $4.46(1.75-11.37) p<0.01$. A logistic regression analysis revealed that obesity is an independent risk factor for second trimester abortion related to $A B I$ with an OR of 3.18 (CI 95\% 1.46-6.91), premature delivery related to $A B I$ with an OR of 2.51 (CI 95\% 1.54-4.09) and for delivery and postpartum infections with an OR of 4.44 (CI 95\% 2.62 to 7.51). Conclusions: Obese pregnant women had a 2.5 to 4.5 times increased risk of infectious morbidity compared to normal weight patients. Obesity is an independent risk factor for second trimester abortion and preterm delivery related to ABI and delivery and postpartum infectious.

(Rev Med Chile 2016; 144: 476-482)

Key words: Obesity; Pregnancy complications, Infectious; Premature Birth.
L a obesidad, definida como índice de masa corporal (IMC) igual o superior a $30 \mathrm{~kg} / \mathrm{m}^{2}$, constituye uno de los problemas más importantes de Salud Pública en el mundo ${ }^{1}$.

Según la Organización Mundial de la Salud (OMS) el sobre peso y la obesidad son el sexto factor de riesgo de defunción en el mundo. Cada año fallecen alrededor 3,4 millones de personas adultas por exceso de peso. Además, $44 \%$ de la carga de diabetes, $23 \%$ de la carga de cardiopatías isquémicas y entre $7 \%$ y $41 \%$ de la carga de algunos cánceres son atribuibles al sobrepeso y la obesidad ${ }^{1}$.

Es bien conocido que la obesidad en el embarazo se asocia con aumento del riesgo de varios resultados adversos del embarazo y parto. Se 
incluyen diabetes gestacional, hipertensión arterial, incremento de las cesáreas, hemorragias del postparto. Adicionalmente son más comunes condiciones fetales como macrosomía, malformaciones congénitas y muerte fetal. Además, los hijos de las madres obesas tienen mayor riesgo que los nacidos de madres de peso normal, de resultados perinatales adversos, como muerte y trauma fetal al nacer, así como una larga estadía en la UCI neonatal ${ }^{2-11}$.

Aunque datos emergentes indican una asociación entre obesidad materna y enfermedades infecciosas ${ }^{12,13}$, hay mucho menos conocimiento disponible. Después de la epidemia de la gripe H1N1, se demostró que las embarazadas obesas tienen mayor riesgo de neumonía viral y peores resultados ${ }^{14,15}$. La obesidad en el embarazo también se ha asociado con un mayor riesgo de infecciones de la piel y de infección de las heridas

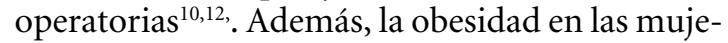
res es un factor de riesgo importante para cistitis y pielonefritis, aunque en los estudios respectivos los autores no separan la condición reproductiva. Por último, algunos estudios han demostrado que la obesidad tiene mayor riesgo de parto prematuro $y$ de endometritis puerperal ${ }^{3,8,13,16-20}$.

El objetivo de este estudio fue comparar los resultados adversos relacionados con infección bacteriana ascendente que presenta la embarazada con índice de masa corporal (IMC) normal y alterado.

\section{Material y Métodos}

\section{Diseño del estudio y base de datos}

Estudio retrospectivo de corte transversal acerca del impacto de la obesidad sobre los resultados adversos relacionados con infección bacteriana ascendente (IBA) en mujeres embarazadas. El Comité de Ética Científico del Servicio de Salud Metropolitano Central aprobó la realización de este trabajo. Los registros clínicos y microbiológicos de las madres y de los recién nacidos se obtuvieron de bases de datos existentes en el Hospital Clínico San Borja Arriarán (HCSBA) que incluyeron: a) Estadísticas anuales del Servicio de Obstetricia, Ginecología y Neonatología (datos generales de todas las embarazadas, infecciones parto, puerperio y neonato); b) Sistema de información perinatal CLAP-OPS/OMS (datos generales, peso y talla maternos); c) Servicio de Laboratorio (datos sobre infecciones parto y puerperio); d) Servicio de Anatomía Patológica (biopsias placentarias de abortos de $2^{\circ}$ trimestre y partos prematuros asociados con infección bacteriana ascendente, IBA); e) Servicio de Neonatología; (morbilidad infecciosa grave del neonato y muerte perinatal asociada a infección).

\section{Población del estudio}

Se estudiaron embarazos únicos entre 15,0 y 42,0 semanas de gestación, atendidos en el HCSBA, entre el 1 de enero de 2012 hasta el 31 de diciembre de 2012. Se excluyeron las siguientes condiciones: aborto menor de 15 semanas, embarazo múltiple, embarazos con malformación fetal, enfermedades que suprimen la respuesta inmune (diabetes pregestacional, VIH, enfermedades del mesenquima), embarazadas sin cálculo del IMC antes de las 11 semanas.

\section{Definiciones de peso corporal}

El IMC se calculó en la primera visita prenatal y antes de las 11 semanas de gestación, de acuerdo con la clasificación de la OMS. Los siguientes grupos se categorizaron: bajo peso (IMC $<18,50$ $\mathrm{kg} / \mathrm{m}^{2}$ ); peso normal (IMC 18,50-24,99 kg/m², grupo de control); sobre peso (IMC 25,00-29,99 $\mathrm{kg} / \mathrm{m}^{2}$ ); obesa clase I (IMC 30,00-34,99 kg/m²); obesa clase II (IMC 35,00-39,99) y obesa clase III $\left(\mathrm{IMC}>40 \mathrm{~kg} / \mathrm{m}^{2}\right)$.

\section{Resultados adversos del embarazo y perinatales asociados con IBA}

Se evaluaron los siguientes resultados adversos asociados con IBA: aborto de segundo trimestre (15-21,6 semanas) y parto prematuro espontáneo entre 22 y 36,6 semanas $^{21-29}$. Infecciones del parto y puerperio: corioamnionitis clínica, infección de la herida operatoria, endometritis y mastitis. Además infección neonatal precoz, incluyendo sepsis, neumonía y muerte perinatal asociada con infección.

Aborto de II trimestre (15-21,6 semanas) y parto prematuro espontáneo (22-36,6 semanas) asociados con IBA. Se consideró aborto o parto prematuro asociado con IBA el embarazo con una o varias de las siguientes condiciones clínicas: rotura prematura de membranas (RPM), infección ovular clínica (corioamnionitis clínica), sangrado vaginal con o sin desprendimiento amniocorial, vaginosis bacteriana sintomática, vaginitis aeróbica, infección del tracto urinario, dispositivo intrauterino 
no extraído, desprendimiento prematuro de placenta normo inserta (DPPNI), cérvix $<$ de $15 \mathrm{~mm}$ medido por ultrasonografía, protrusión de membranas ovulares por el cérvix y asociados con uno o varios de los siguientes hallazgos histopatológicos placentarios: corioamnionitis, funisitis, intervellositis, hematoma y hemorragia retroplacentaria ${ }^{21-30}$.

Infecciones parto, puerperio. Corioamnionitis clínica, diagnóstico realizado según criterios propuestos por Gibbs y asociados ${ }^{30}$. Infección herida operatoria: inflamación local herida operatoria de cesárea con drenaje sero-hemo/purulento o drenaje de absceso o resutura de laparotomía o de episiorrafia dehiscentes. Endometritis: puérpera con una o varias de las siguientes condiciones clínicas, dolor abdominal, metrorragia, temperatura axilar $>38,0{ }^{\circ} \mathrm{C}$, sensibilidad uterina, descarga vaginal turbia o de mal olor, leucocitosis materna $\left(>15.000\right.$ cels $\left./ \mathrm{mm}^{3}\right)$. Mastitis: puérpera con inflamación local mamaria y elevación temperatura axilar $>38,0^{\circ} \mathrm{C}$ o más o con infección circunscrita y microorganismo aislado por punción o drenaje quirúrgico o con absceso mamario y drenaje quirúrgico ${ }^{31}$.

Infección neonatal precoz grave (primeros 7 días de vida ${ }^{21,22}$. Neumonía, compromiso inflamatorio agudo alveolar, intersticial y de vía aérea con hallazgos radiológicos compatibles, de origen intrauterino, que se presenta en el feto (congénita) y/o neonato (connatal) dentro de las $24 \mathrm{~h}$ de vida. Sepsis, neonato con cuadro multisistémico clínicamente compatible con infección y con hemocultivos positivos. Muerte perinatal asociada a infección del feto-neonato o por DPPNI o prematurez extrema por parto prematuro por IBA ${ }^{22}$.

\section{Análisis estadístico de los datos y expresión de resultados}

Se usó análisis bivariado para comparar las tasas de, aborto de $2^{\circ}$ trimestre y parto prematuro asociados con IBA, infección del parto, puerperio e infección neonatal precoz y mortalidad perinatal, en función del IMC dividido en categorías normal y obesa. Los resultados se expresaron como OR \pm IC de 95\%. Se usó regresión logística binaria para el análisis de las tasas de, aborto de $2^{\circ}$ trimestre y parto prematuro asociados con IBA, infección del parto, puerperio e infección neonatal precoz y mortalidad perinatal, teniendo como variables independientes edad materna, paridad y obesidad. Los resultados se expresaron como OR \pm IC de $95 \%$.

\section{Resultados}

Un total de 6.150 mujeres tuvieron parto o aborto de $2^{\circ}$ trimestre en la Maternidad del HCSBA durante 2012. De ellas 5.919 (96,2\%) cumplieron con los criterios de inclusión (5.870 partos y 49 abortos de II trimestre). La Figura 1 muestra el diagrama de flujo de la incorporación de pacientes al estudio y las razones de la exclusión. Se excluyeron 231 embarazadas: 85 debido a embarazos múltiples, 68 por falta de talla y/o peso en la historia clínica, 53 debido a malformaciones fetales y 25 casos por enfermedades inmunosupresoras ( 15 diabetes preexistente, $9 \mathrm{VIH}+$ y 1 con enfermedad del mesénquima).

\section{Características de la población en estudio}

La Tabla 1 contiene datos de la edad media $( \pm)$, paridad, tipo de parto y la incidencia de resultados

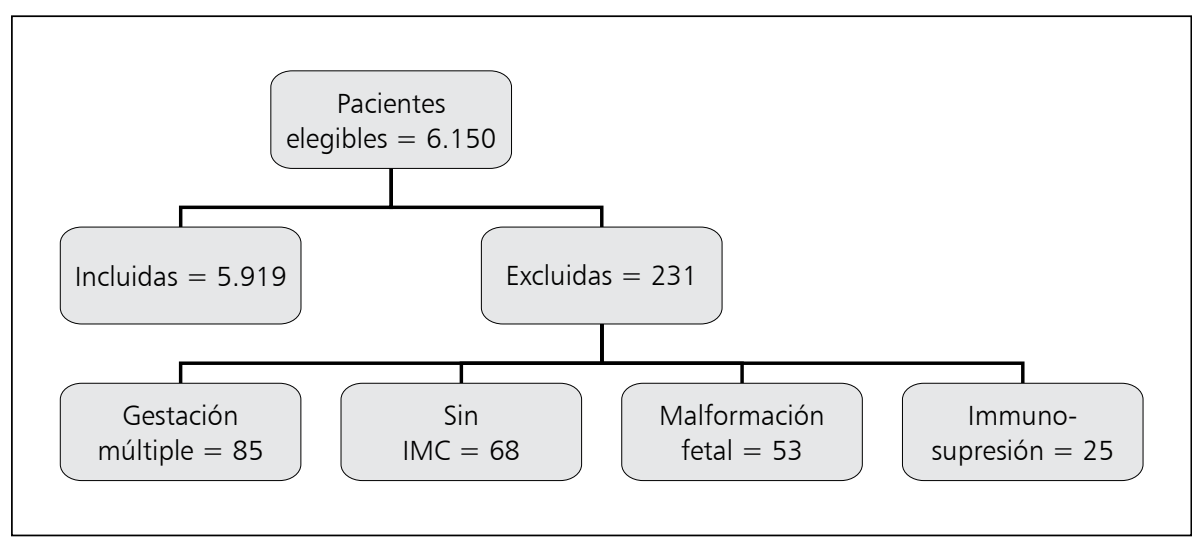

Figura 1. Diagrama de flujo. Pacientes incorporadas al estudio. 
Tabla 1. Características de la población en estudio. Hospital San Borja Arriarán 2012

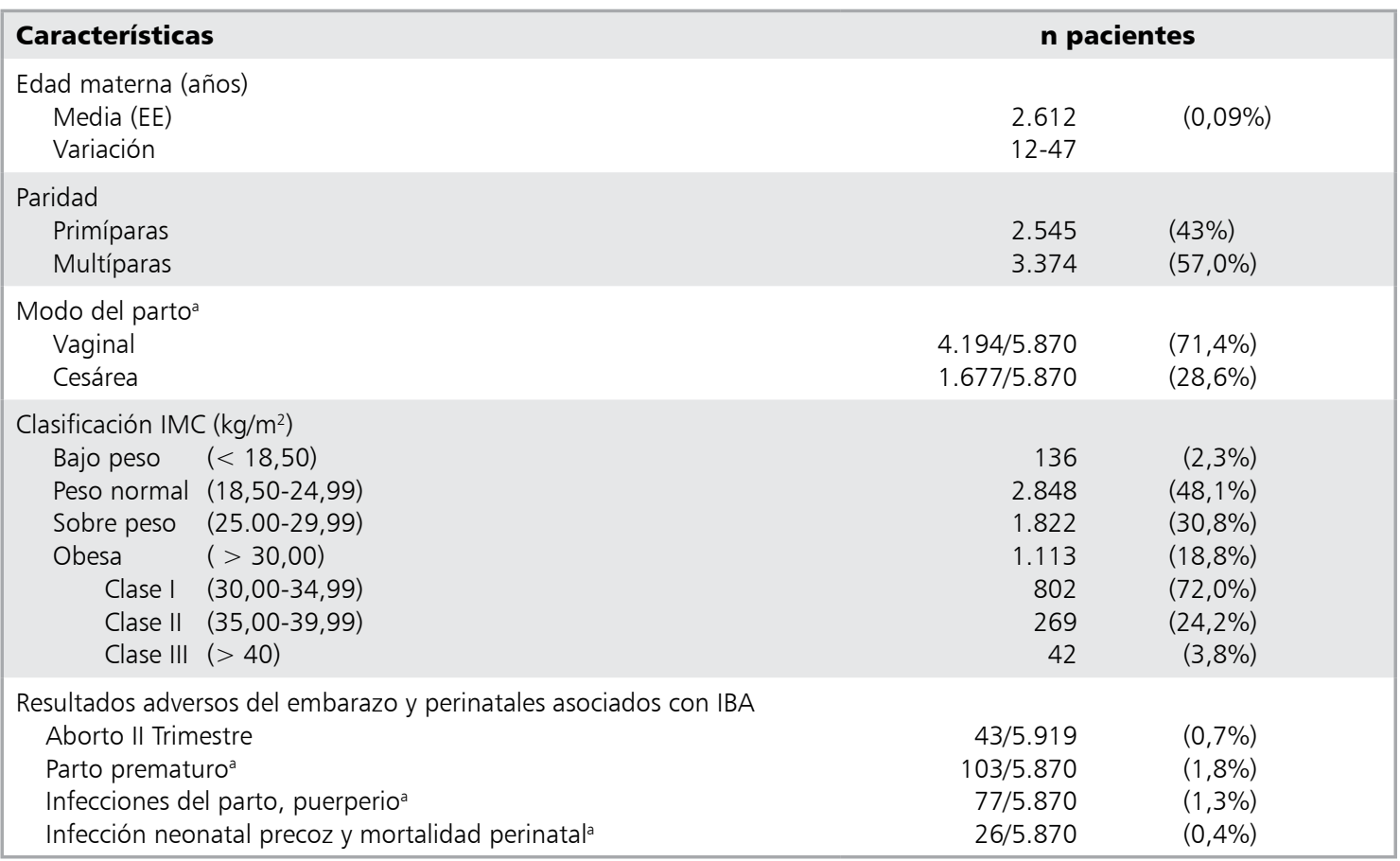

aSe restaron 49 abortos de II trimestre. EE: Error estándar. IMC: Índice de masa corporal. IBA: Infección bacteriana ascendente.

adversos del embarazo y perinatales asociados con IBA en la población estudiada. Infecciones del parto y puerperio se encontraron en 77 mujeres: corioamnionitis clínica 33, infección herida operatoria 28 , endometritis puerperal 5 , mastitis 5 , infección del tracto urinario (ITU) 5, peritonitis 1. Complicaciones perinatales asociadas a IBA se encontraron en 26 casos, muertes fetales 12: por hipoxia aguda secundaria a DPPNI por IBA 7 , por hipoxia aguda por funisitis 5 ; muertes neonatales 7: por sepsis 2, por neumonía 1, por prematurez extrema 4 asociada a parto prematuro por IBA; morbilidad severa 7, todas neumonías connatales.

De acuerdo con las definiciones de la OMS IMC, se clasificaron las embarazadas como: bajo peso $(\mathrm{n}=136,2,3 \%)$, peso normal $(\mathrm{n}=2.848$, $48,1 \%)$, sobre peso $(\mathrm{n}=1.822,30,8 \%)$ y obesas $(\mathrm{n}=1.113,18,8 \%)$.

\section{Resultados adversos del embarazo y perinatales asociados con IBA según IMC}

Los siguientes resultados adversos se presentaron más frecuentemente en pacientes obesas que en aquellas con peso normal: aborto II trimestre asociado con IBA $1,4 \%$ vs $0,4 \%$, OR 3,45 (95\% IC 1,63-7,31); parto prematuro asociado con IBA $3,1 \%$ vs $1,3 \%$, OR 2,42 (95\% IC $1,51-3,87)$; infecciones del parto y puerperio $3,2 \%$ vs $1,0 \%$, OR 3,42 (95\% IC 2,06-5,68) e infección neonatal precoz y mortalidad perinatal asociadas con IBA $1,1 \%$ vs $0,2 \%$, OR 4,463 (95\% IC $1,75-11,37$ ) (Tabla 2).

Los resultados adversos del embarazo asociados con IBA en pacientes con sobre peso no fueron significativamente diferentes a los encontrados en controles.

El análisis de regresión logística reveló que la obesidad en el embarazo es factor de riesgo de aborto II trimestre asociado con IBA, OR 3,18 (IC 95\% 1,46-6,91), de parto prematuro asociado con IBA OR 2,51 (IC 95\% 1,54-4,09) y de infecciones del parto y puerperio OR 4,44 (IC 95\% 2,62-7,51) independiente de la edad y de la paridad materna. El efecto de la multiparidad sobre infecciones del parto-puerperio y perinatales, no se puede determinar en presencia de edad y obesidad, porque estas variables pueden estar correlacionadas entre sí (Tabla 3). 
Tabla 2. Infecciones asociadas con IBA durante el embarazo en pacientes con peso normal y obesas. Hospital San Borja Arriarán 2012

\begin{tabular}{|c|c|c|c|c|}
\hline $\begin{array}{l}\text { Resultados adversos del embarazo y } \\
\text { perinatales asociados con IBA }\end{array}$ & $\begin{array}{l}\text { Pacientes con } \\
\text { peso normal } \\
n=2.848\end{array}$ & $\begin{array}{c}\text { Pacientes } \\
\text { obesas } \\
n=1.113\end{array}$ & $\mathbf{p}$ & OR (95\% IC) \\
\hline Aborto II trimestre & $12 / 2.848(0,4 \%)$ & $16 / 1.113(1,4 \%)$ & 0,001 & $3,45(1,63-7,31)$ \\
\hline Parto prematuro & $37 / 2.832(1,3 \%)$ & $34 / 1.097(3,1 \%)$ & 0,0001 & $2,42(1,51-3,87)$ \\
\hline Infecciones del parto, puerperio ${ }^{a}$ & $27 / 2.832(1,0 \%)$ & $35 / 1.097(3,2 \%)$ & 0,0001 & $3,42(2,06-5,68)$ \\
\hline Infección neonatal precoz y mortalidad perinatal ${ }^{a}$ & $7 / 2.832(0,2 \%)$ & $12 / 1.097(1,1 \%)$ & 0,001 & $4,46(1,75-11,37)$ \\
\hline
\end{tabular}

IBA: Infección bacteriana ascendente. ${ }^{a}$ Se restaron 32 abortos de $2^{\circ}$ trimestre.

Tabla 3. Análisis de regresión logística de los factores de riesgo para infecciones durante el embarazo. Hospital San Borja Arriarán 2012

\begin{tabular}{|lccc|}
\hline $\begin{array}{l}\text { Variables dependientes } \\
\text { Resultados adversos del embarazo y } \\
\text { perinatales asociados con IBA }\end{array}$ & $\begin{array}{c}\text { Edad materna } \\
\text { OR (95\%IC) }\end{array}$ & $\begin{array}{c}\text { Paridad } \\
\text { OR (95\%IC) }\end{array}$ & $\begin{array}{c}\text { Obesidad } \\
\text { OR (95\%IC) }\end{array}$ \\
\hline Aborto II trimestre & $1,01(0,94-1,08)$ & $1,36(0,54-3,40)$ & $3,18(1,46-6,91)$ \\
\hline Parto prematuro & $1,02(0,98-1,06)$ & $0,60(0,34-1,04)$ & $2,51(1,54-4,09)$ \\
\hline Infecciones del parto, puerperio & $1,02(0,97-1,06)$ & $0,21(0,11-0,40)$ & $4,44(2,62-7,51)$ \\
\hline Infección neonatal precoz y mortalidad perinatal & $1,12(1,04-1,20)$ & $0,31(0,11-0,89)$ & $3,9(1,49-10,21)$ \\
\hline
\end{tabular}

IBA: Infección bacteriana ascendente.

\section{Discusión}

La obesidad constituye un serio problema de salud pública en el mundo. En este estudio, la prevalencia de la obesidad y el sobrepeso en las mujeres atendidas en un hospital público (HCSBA) fueron $19 \%$ y $31 \%$, respectivamente.

Durante el embarazo la obesidad está relacionada con diabetes gestacional, hipertensión, preeclampsia, macrosomía, hemorragia posparto, aumento de partos por cesárea, trauma fetal al nacer y malformaciones congénitas, que empeoran los riesgos perinatales ${ }^{2-11}$.

Sin embargo, muy pocas publicaciones se han centrado en investigar el efecto de la obesidad sobre las complicaciones infecciosas en el embarazo, parto y puerperio $3,8,10,11-15$.

En nuestro estudio, la obesa embarazada tiene un riesgo 2,5 a 4,5 veces más alto que las mujeres de peso normal de presentar resultados adversos del embarazo asociados con IBA: aborto de II trimestre, parto prematuro, infecciones del parto- puerperio e infección neonatal precoz y mortalidad perinatal por infección. Además de- mostramos que la obesidad es un factor de riesgo independiente de aborto de II trimestre por IBA, de parto prematuro por IBA y de infecciones del parto y puerperio. En pacientes con sobre peso estas consecuencias no fueron diferentes a las encontradas en mujeres controles.

Estos resultados son de gran importancia ya que identificar la obesidad como factor de riesgo de resultados adversos por IBA durante el embarazo, permite tomar las medidas adecuadas para evitarlos. Especial relevancia adquiere la pesquisa y el tratamiento de las infecciones vaginales (vaginosis bacteriana, vaginitis aeróbica) en embarazadas obesas para prevenir su ascenso y el parto prematuro consiguiente, ya que la tasa de prematurez por esta causa ha aumentado en nuestro hospital ${ }^{12,22}$ $y$ en todo el mundo ${ }^{23}$. El conocimiento del riesgo de infección que tiene la embarazada obesa permite que sean más eficientes las intervenciones tendientes a reducir estos resultados adversos ${ }^{21,22}$.

También surge la inquietud de conocer el origen de la mayor frecuencia de infecciones en la gestante con obesidad. Se sabe que en el embarazo la obesa tiene alteración de la inmunidad celular. 
Posee una proporción significativamente menor de linfocitos CD8 y de células T NK, una mayor proporción de células $\mathrm{B}$, producción alterada de citoquinas y deterioro de la capacidad de proliferación de los linfocitos ${ }^{32}$. La obesidad lleva hacia un estado proinflamatorio. La inflamación del tejido adiposo está acompañada con un aumento de los niveles circulantes de citoquinas proinflamatorias (TNF- $\alpha$, IL-6) y proteínas de fase aguda (PCR, fibrinógeno, haptoglobina), en obesas embarazadas y no embarazadas ${ }^{33}$. La mayoría de estos productos proinflamatorios es secretada en la circulación por los adipocitos y macrófagos, y puede ser en parte responsable de la inflamación sistémica ${ }^{34}$. Es posible que el estado inflamatorio crónico de los obesos evite el funcionamiento apropiado de la respuesta inmune y promueva el desarrollo de infecciones.

El sistema inmune innato vaginal representa la primera línea de defensa contra patógenos externos y mantiene en equilibrio la microbiota comensal oportunista, impidiendo el ascenso de microorganismos al tracto genital superior. Es probable que la respuesta inmune innata en embarazadas obesas esté deteriorada en la vagina ${ }^{35,36}$. Estas alteraciones de la inmunidad permitirían explicar la mayor frecuencia de aborto de II trimestre, parto prematuro, infecciones del parto, puerperio e infecciones perinatales producto de infecciones que se inician en la vagina. Futuros trabajos se requieren en embarazadas obesas para demostrar este posible deterioro de la inmunidad innata de la vagina.

La limitación de este estudio fue el sesgo producido por la falta de resultados de infección vaginal e ITU. Los datos incompletos del Laboratorio del HCSBA y la imposibilidad de revisar las fichas clínicas de las pacientes por falta de consentimiento informado, nos impidió presentar estos datos.

Los resultados de este trabajo concluyen que la mujer obesa tiene significativo mayor riesgo que la de peso normal de presentar resultados adversos en el embarazo y perinatales asociados con IBA. Además la obesidad es factor de riesgo independiente de aborto de II trimestre por IBA, de parto prematuro por IBA y de infecciones del parto y puerperio. El conocimiento del riesgo de infección que tiene la obesa permite introducir intervenciones oportunas en el embarazo tendientes a reducir el parto prematuro y la morbimortalidad perinatal asociada.

\section{Referencias}

1. OMS. Obesidad y sobrepeso. Centro de prensa. Nota descriptiva N³11. Mayo de 2014.

2. Scott-Pillai R, Spence D, Cardwell CR, Hunter A, Holmes VA. The impact of body mass index on maternal and neonatal outcomes: a retrospective study in a UK obstetric population, 2004-2011. BJOG 2013; 120: 932-9.

3. Heslehurst N, Simpson H, Ells LJ, Rankin J, Wilkinson J, Lang R, et al. The impact of maternal BMI status on pregnancy outcomes with immediate short-term obstetric resource implications: a meta-analysis. Obes Rev 2008; 9: 635-83.

4. Leung TY, Leung TN, Sahota DS, Chan OK, Chan LW, Fung TY, et al. Trends in maternal obesity and associated risks of adverse pregnancy outcomes in a population of Chinese women. BJOG 2008; 115: 1529-37.

5. Gunatilake RP, Smrtka MP, Harris B, Kraus DM, Small MJ, Grotegut CA, et al. Predictors of failed trial of labor among women with an extremely obese body mass index. Am J Obstet Gynecol 2013; 209: 562.e1-5.

6. Cai GJ, Sun XX, Zhang L, Hong Q. Association between maternal body mass index and congenital heart defects in offspring: a systematic review. Am J Obstet Gynecol 2014; 211: 91-117.

7. Ouzounian JG. Risk factors for neonatal brachial plexus palsy. Semin Perinatol 2014; 38: 219-21.

8. Stamilio DM, Scifres CM. Extreme obesity and postcesarean maternal complications. Obstet Gynecol 2014; 124: 227-32.

9. Yao R, Ananth CV, Park BY, Pereira L, Plante LA. Perinatal Research Consortium. Obesity and the risk of stillbirth: a population-based cohort study. Am J Obstet Gynecol 2014; 210: 457.e1-9.

10. Leth RA, Uldbjerg N, Nørgaard M, Møller JK, Thomsen RW. Obesity, diabetes, and the risk of infections diagnosed in hospital and post-discharge infections after cesarean section: a prospective cohort study. Acta Obstet Gynecol Scand 2011; 90: 501-9.

11. Sebire NJ, Jolly M, Harris JP, Wadsworth J, Joffe M, Beard RW, et al. Maternal obesity and pregnancy outcome: a study of 287,213 pregnancies in London. Int J Obes Relat Metab Disord 2001; 8: 1175-82.

12. Tipton AM, Cohen SA, Chelmow D. Wound infection in the obese pregnant woman. Semin Perinatol 2011; 35: 345-9.

13. Huttunen R, Syrjänen J. Obesity and the risk and outcome of infection. Int J Obes (Lond) 2013; 37: 333-40.

14. Fezeu L, Julia C, Henegar A, Bitu J, Hu FB, Grobbee DE, et al. Obesity is associated with higher risk of intensive 
care unit admission and death in influenza A ( $\mathrm{H} 1 \mathrm{~N} 1)$ patients: a systematic review and meta-analysis. Obes Rev 2011; 12: 653-9.

15. Maraví-Poma E, Martin-Loeches I, Regidor E, Laplaza C, Cambra K, Aldunate S, et al; Grupo Español de Trabajo de Gripe Grave A (SEMICYUC). Severe 2009 A/ H1N1v influenza in pregnant women in Spain. Crit Care Med 2011; 39: 945-51.

16. Karppelin M, Siljander T, Vuopio-Varkila J, Kere J, Huhtala H, Vuento R, et al. Factors predisposing to acute and recurrent bacterial non-necrotizing cellulitis in hospitalized patients: a prospective case-control study. Clin Microbiol Infect 2010; 16: 729-34.

17. Paiva LV, Nomura RM, Dias MC, Zugaib M. Maternal obesity in high-risk pregnancies and postpartum infectious complications. Rev Assoc Med Bras 2012; 58: 453-8.

18. Lynch AM, Hart JE, Agwu OC, Fisher BM, West NA, Gibbs RS. Association of extremes of prepregnancy BMI with the clinical presentations of preterm birth. Am J Obstet Gynecol 2014; 210: 428.e1-9.

19. Cnattingius S, Villamor E, Johansson S, Edstedt Bonamy AK, Persson M, Wikström AK, et al. Maternal obesity and risk of preterm delivery. JAMA 2013; 309: 2362-70.

20. Shaw GM, Wise PH, Mayo J, Carmichael SL, Ley C, Lyell DJ, et al, March of Dimes Prematurity Research Center at Stanford University School of Medicine. Maternal prepregnancy body mass index and risk of spontaneous preterm birth. Paediatr Perinat Epidemiol 2014; 28: 30211.

21. Ovalle A, Kakarieka E, Rencoret G, Fuentes A, del Río MJ, Morong C, et al. Factores asociados con el parto prematuro entre 22 y 34 semanas en un hospital público de Santiago. Rev Med Chile 2012; 140: 19-29.

22. Ovalle A, Kakarieka E, Díaz M, García Huidobro T, Acuña MJ, Morong K, et al. Mortalidad perinatal en el parto prematuro entre 22 y 34 semanas en un hospital público de Santiago, Chile. Rev Chil Obstet Ginecol 2012; 77: 263-70.

23. MacDorman MF, Mathews TJ. Birth Stats: percentage of preterm births, United States and selected European countries, 2004. Birth 2010; 37: 168.

24. Gómez R, Romero R, Nien JK, Medina L, Carstens M, Kim YM, et al. Idiopathic vaginal bleeding during pregnancy as the only clinical manifestation of intrauterine infection. J Matern Fetal Neonatal Med 2005; 18: 31-7.

25. Madan I, Romero R, Kusanovic JP, Mittal P, Chaiwo- rapongsa $\mathrm{T}$, Dong $\mathrm{Z}$, et al. The frequency and clinical significance of intra-amniotic infection and/or infl ammation in women with placenta previa and vaginal bleeding: an unexpected observation. J Perinat Med 2010; 38: 275-9.

26. Hassan S, Romero R, Hendler I, Gómez R, Khalek N, Espinoza $\mathrm{J}$, et al. A sonographic short cérvix as the onlyclinical manifestation of intra-amniotic infection. J Perinat Med 2006; 34: 13-9.

27. Goldenberg RL, Andrews WW, Faye-Petersen O, Cliver S, Goepfert AR, Hauth JC. The Alabama Preterm Birth Project: placental histology in recurrent spontaneous and indicated preterm birth. Am J Obstet Gynecol 2006; 195: 792-6.

28. Ovalle A, Kakarieka E, Correa A, Vial MT, Aspillaga C. Estudio anátomo-clínico de las causas de muerte fetal. Rev Chil Obstet Ginecol 2005; 70: 303-12.

29. Ovalle A, Gómez R, Martínez MA, Kakarieka E, Fuentes A, Aspillaga C, et al. Invasión microbiana de la cavidad amniotica en la rotura de membranas de pretérmino Resultados maternoneonatales y patología placentaria según microorganismo aislado. Rev Med Chile 2005; 133: 51-61.

30. Gibbs RS, Castillo MS, Rodgers PJ. Management of acute chorioamnionitis. Am J Obstet Gynecol 1980; 136: 709-13.

31. Murray PR, Baron EJ, Jorgenssen JH, Landry ML, Pfaller MA (Eds.), "Manual of Clinical Microbiology", 9th edition, Washington D.C., ASM Press, 2007.

32. Sen S, Iyer C, Klebenov D, Histed A, Aviles JA, Meydani SN. Obesity impairs cell-mediated immunity during the second trimester of pregnancy. Am J Obstet Gynecol 2013; 208: 139.e1-8.

33. Ramsay JE, Ferrell WR, Crawford L, Wallace AM, Greer IA, Sattar N. Maternal obesity is associated with dysregulation of metabolic, vascular, and inflammatory pathways. J Clin Endocrinol Metab 2002; 87: 4231-7.

34. Fain JN. Release of inflammatory mediators by human adipose tissue is enhanced in obesity and primarily by the nonfat cells: a review. Mediators Inflamm 2010; 2010; 513948: 1-20.

35. Witkin SS, Linhares IM, Giraldo P, Ledger WJ. An altered immunity hypothesis for the development of symptomatic bacterial vaginosis. Clin Infect Dis 2007; 44 (4): 554-7.

36. Hedges SR, Barrientes F, Desmond RA, Schwebke JR. Local and systemic cytokine levels in relation to changes in vaginal flora. J Infect Dis 2006; 193: 556-62. 